\title{
An integrated vapor source with a porous silicon wick
}

\author{
J. Z. Wallner ${ }^{*}, 1,2$, K. S. Kunt ${ }^{2}$, H. Obanionwu ${ }^{2}$, M. C. Oborny ${ }^{2,3}$, P. L. Bergstrom ${ }^{1,2}$, \\ and E. T. Zellers ${ }^{2}$ \\ ${ }^{1}$ Department of Electrical and Computer Engineering, Michigan Technological University, \\ 1400 Townsend Drive, Houghton, MI 49931-1295, USA \\ 2 Engineering Research Center for Wireless Integrated MicroSystems, the University of Michigan, \\ Ann Arbor, MI, USA \\ ${ }^{3}$ Sandia National Laboratories, Albuquerque, NM, USA
}

Received 17 March 2006, revised 15 September 2006, accepted 15 November 2006 Published online 19 April 2007

PACS 51.20. $+\mathrm{d}, 85.85 .+\mathrm{j}$

A micro vapor source has been developed for calibrating micro gas chromatograph $(\mu \mathrm{GC})$ systems. By utilizing a porous silicon wick in a micro diffusion system, vapor generation with excellent stability has been achieved. The source has shown uniform and repeatable vapor generation for n-decane with less than a $0.1 \%$ variation in 9 hours, and less than a $0.5 \%$ variation in rate over 7 days. The evolution rate follows the diffusion model as expected, although the room temperature rate is higher than theoretically predicted. Equipped with a refillable reservoir, this vapor source is suitable for extended $\mu \mathrm{GC}$ field deployment.

() 2007 WILEY-VCH Verlag GmbH \& Co. KGaA, Weinheim

\section{Introduction}

Gas chromatography (GC) is the most widely used method for analyzing mixtures of volatile and semivolatile organic compounds. However, the bulkiness of the typical laboratory GC system hinders its application in fields that require low cost on-site analysis, such as environmental monitoring. In a worldwide effort to miniaturize GC system for the sake of better portability, faster response time, potentially higher performance, lower dead volume, lower power consumption and lower cost, MEMS based $\mu \mathrm{GC}$ instruments are being developed at several national laboratories and universities [1-5].

Although it is possible to do on-site analysis of air samples for environmental monitoring with the emerging $\mu \mathrm{GC}$ systems, the accuracy of $\mu \mathrm{GC}$ measurement still relies on the frequent calibration with gaseous standards, which involves the generation of standard atmospheres and injection of a standard atmosphere into the $\mu \mathrm{GC}$. In order to simplify the calibration procedure, micro calibration vapor sources have been developed for the $\mu \mathrm{GC}$ system. By automatically adding vapors with known concentration (internal standard) into air samples, the integrated calibration source provides a means for collecting realtime diagnostic information to compensate quantitative and qualitative errors caused by sampling and instrument drift.

In our previous work, controllable vapor generation has been achieved by a three-layer diffusion device consisting of a porous silicon (PS) reservoir, a spacer, and a cap with a micromachined diffusion channel and an outlet port [6]. However, that design has two drawbacks. Because the porous silicon reservoir has to be filled before the device is put together, room temperature polymer-based bonding is the only choice for device assembly. Unfortunately, those polymer-based bonds not only introduce contaminative vapors, but also suffer deterioration in the presence of the calibrant liquid, such as n-decane.

* Corresponding author: e-mail: jizheng@mtu.edu, Phone: +1 906487 2550, Fax: +1 9064872949 
Secondly, since the porous silicon reservoir cannot be refilled after assembly, the vapor source's lifetime is limited by the depth of the porous silicon layer and the storage time before utilization. In this paper, we present a new design of the integrated calibration source using a PS wick and an external reservoir to improve its manufacturability, system integration, and to extend its lifetime.

\section{Theory}

The new design of the integrated calibration source is shown in Fig. 1. It consists of three layers. The top layer has a deep recess and a through hole, which serves as the reservoir and the filling port of the calibration source. The middle layer is a macro porous silicon membrane, which serves as a wick for the reservoir. The bottom layer has a shallow recess of the same size of the PS wick, a groove and a small hole in it. When the device is assembled, the shallow recess will form a headspace over the PS wick; the groove will form a diffusion channel; and the small hole becomes an outlet port for the diffusion channel. In operation, the reservoir will be filled with calibrant liquid through the filling port. The PS wick holds the calibrant liquid by capillary forces while allowing the calibrant vapor to enter into the headspace. The calibrant vapor then transports through the attached diffusion channel and the outlet port into the sample inlet of the $\mu \mathrm{GC}$, where the calibrant vapor will mix with the carrier gas stream. Two equations describe the whole process. The first one is the Kelvin equation, which gives the vapor pressure $\left(P_{\mathrm{v}}\right)$ of the calibrant liquid held by porous media:

$$
\ln \left(\frac{P_{\mathrm{v}}}{P_{\text {sat }}}\right)=\frac{2 \gamma V_{1}}{R T r},
$$

where $P_{\text {sat }}$ is the saturation vapor pressure, $\gamma$ is the surface tension of the liquid, $V_{1}$ is the molar volume of the liquid, $R$ is the molar gas constant, $T$ is the absolute temperature and $r$ is the liquid radius of curvature. For the liquid held by capillary force, $r$ approximates to the radius of the pore size. The second equation is the diffusion equation, which describes the transportation of the calibrant vapor from the headspace cavity into the sample inlet of the $\mu \mathrm{GC}$ through the diffusion channel. The calibration source's generation rate $q_{\mathrm{d}}$ can be calculated according to the diffusion equation:

$$
q_{\mathrm{d}}=D M P A \ln \left(\frac{P}{P-P_{\mathrm{v}}}\right) / L R T,
$$

where $D$ is the vapor diffusion coefficient, $M$ is the vapor molecular weight, $A$ is the diffusion channel cross section, $P$ is the atmospheric pressure, and $L$ is the diffusion channel length. At constant atmospheric pressure, the generation rate of the calibration source can be adjusted by changing the diffusion channel's dimension or the device's operation temperature.

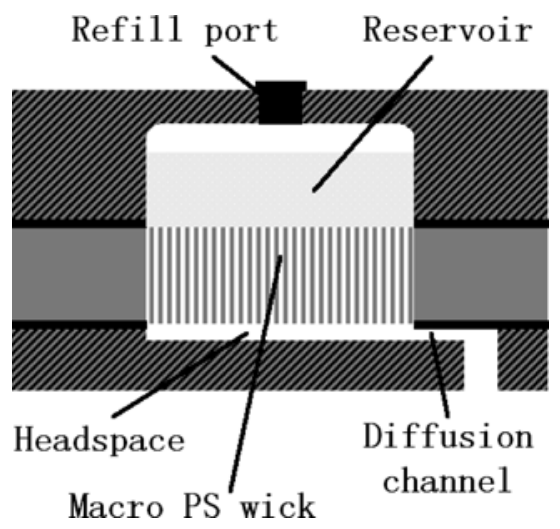

C) 2007 WILEY-VCH Verlag GmbH \& Co. KGaA, Weinheim
Fig. 1 Schematic of the integrated calibration source with a macro porous silicon wick. 


\section{Experimental results and discussion}

MEMS technology has been used to fabricate the calibration vapor source. Both the reservoir layer and the diffusion channel layer are made from \#7740 Pyrex glass using micromilling [7]. The reservoir has a capacity of $6.7 \mu \mathrm{L}$. Diffusion channels of three different lengths $(7.5,13$ and $20.5 \mathrm{~mm})$ and a crosssection of $200 \times 200 \mu \mathrm{m}^{2}$ have been fabricated. The porous silicon wick is made from p-type (100) substrates with resistivity of $17-23 \Omega \mathrm{cm}$. The PS region has a diameter of $3.5 \mathrm{~mm}$, defined by a $\mathrm{SiO}_{2} / \mathrm{Si}$ double layer mask fabricated by RF sputter deposition. Standard photolithography and subsequent $\mathrm{KOH}$ etching were used to form arrays of inverted pyramid pits as initial pores. An aqueous electrolyte with $10^{-3} \mathrm{M}$ cetyltrimethylammonium chloride (CTAC) and $\mathrm{HF}(49 \%)$, ethanol, $\mathrm{H}_{2} \mathrm{O}$ at volume ratio $1: 2: 3$ was used for the PS formation. Anodization was performed at a current density of $27 \mathrm{~mA} / \mathrm{cm}^{2}$. Throughwafer pores ( $>500 \mu \mathrm{m}$ long) were obtained by simply stacking a sacrificial sample at the backside of the macroporous sample during the anodization. Details for the through-wafer pore formation have been reported previously [8]. The surface and the cross section of the macro porous membrane are shown in Fig. 2. The device was then assembled by In-Au bonding, which provides a uniform hermetic seal, unlike polymer bonding, without introducing organic contamination. The final devices are shown in Fig. 3.

For testing, the source was loaded with n-decane and connected to a standard GC-FID (Flame Ionization Detector) through a custom-built polyetheretherketone (PEEK $\left.{ }^{\mathrm{TM}}\right)$ fixture. The experimental setup is shown in Fig. 4. Reproducibility of the vapor generation rate has been tested over several weeks at constant temperatures. Small variations of $0.1 \%$ over a 9 hour period and $0.5 \%$ over a week have been observed, as shown in Fig. 5(a). Repeatable results were obtained between refills of the reservoir.

The excellent stability of the vapor generation rate owes much to the macro porous silicon wick. According to Eq. (1), the calibrant vapor pressure in the headspace is related with the pore size of the porous wick. The impact of pore sizes on the vapor pressure is shown in Fig. 6. When the pore diameter is less than $0.5 \mu \mathrm{m}$, a small variation in pore size will result in a significant variation in the vapor pressure. As the pore size increases, the impact of pore size on the vapor pressure reduces. In our experiment, the macro porous silicon wick has a pore size of $3.5 \mu \mathrm{m}$, which can provide sufficient capillary force without having a huge impact on the vapor pressure. In addition, the pore size is very uniform along the full
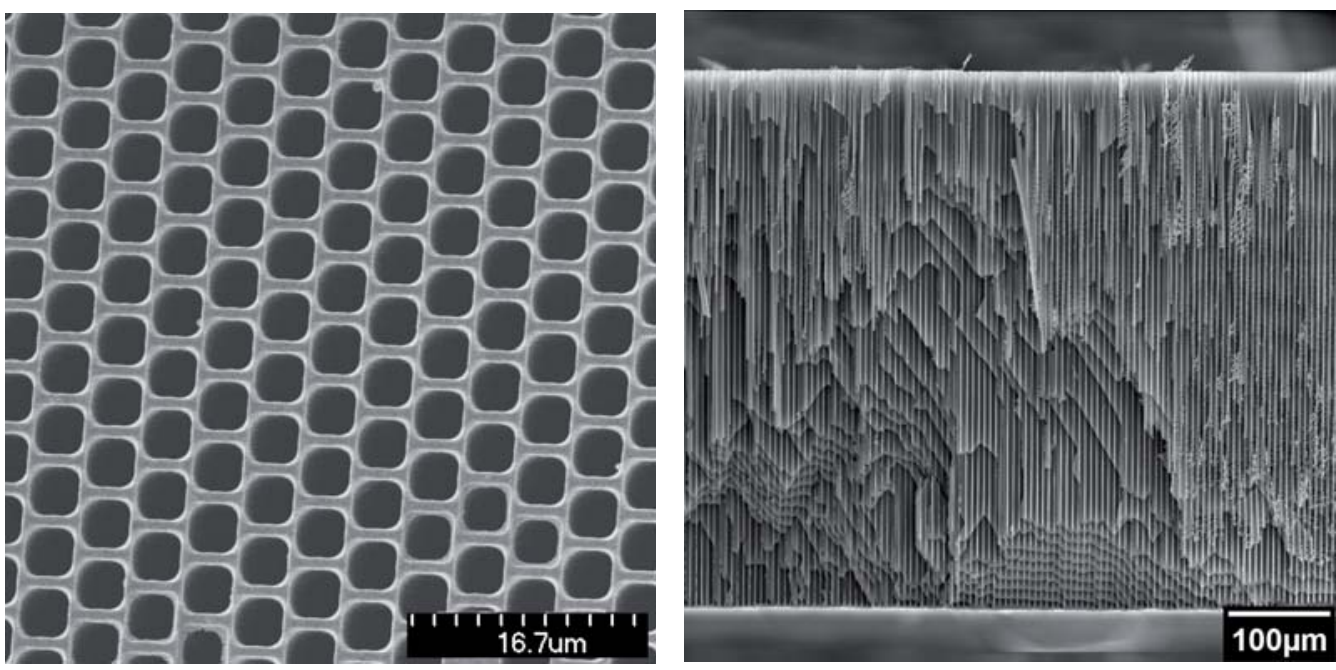

(a) (b)

Fig. 2 (a) Top view of the macro porous silicon wick; (b) cross sectional view of the macro porous silicon wick. 


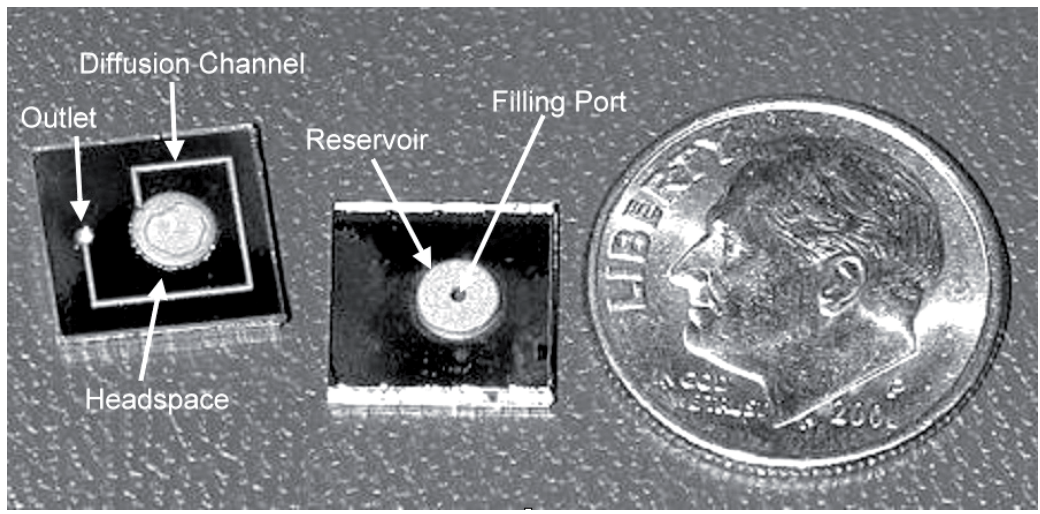

Fig. 3 Picture of two assembled calibration sources, showing the reservoir, the filling port, the headspace, the diffusion channel, and the outlet port.

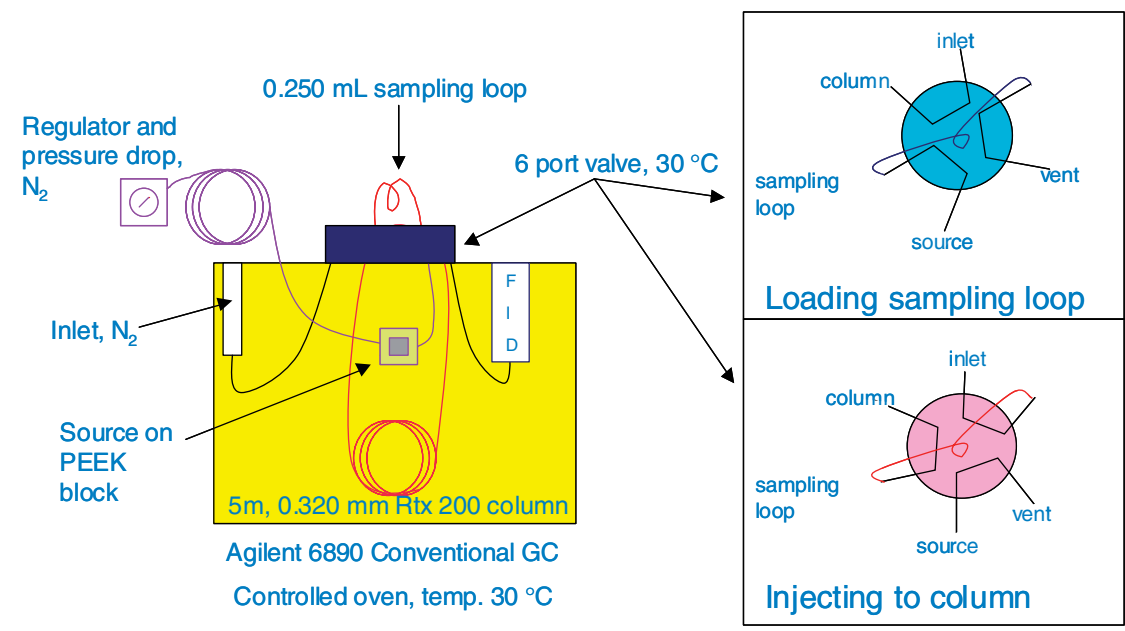

Fig. 4 (online colour at: www.pss-a.com) Experimental setup for testing the vapor calibration source.
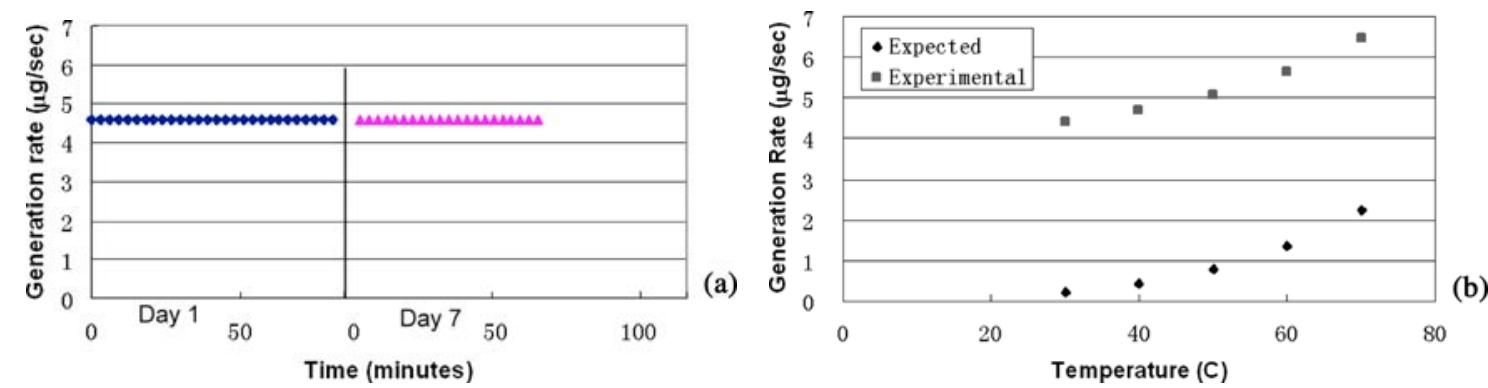

Fig. 5 (online colour at: www.pss-a.com) (a) Steady-state generation rate at $30^{\circ} \mathrm{C}$; (b) temperature dependence of generation rate for $30-70{ }^{\circ} \mathrm{C}$. 

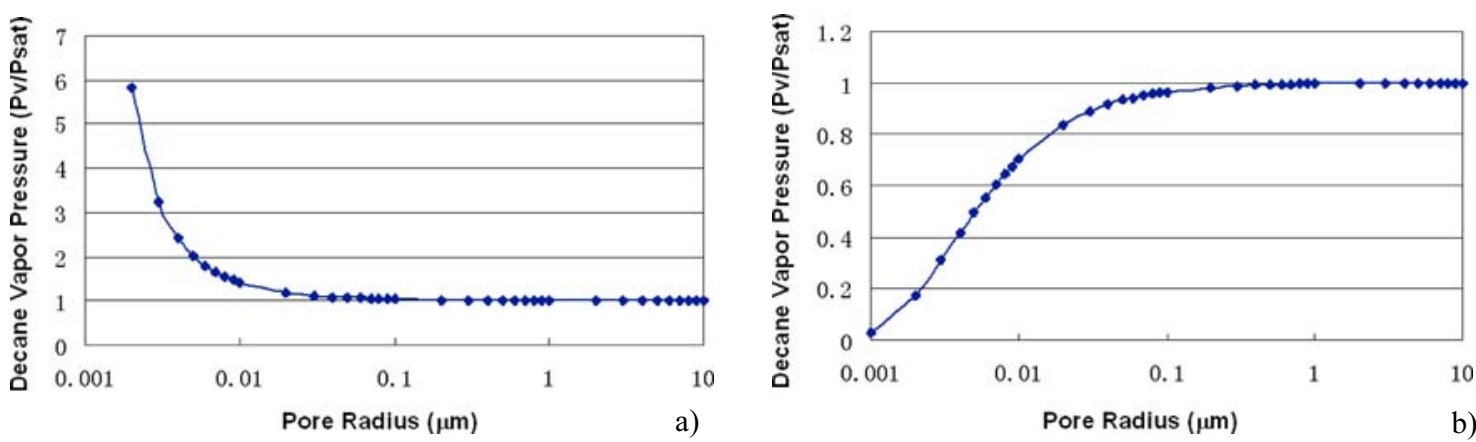

Fig. 6 (online colour at: www.pss-a.com) Impact of pore size on the vapor pressure with (a) convex liquid surface; (b) concave liquid surface.

thickness of the PS wick. Therefore, a constant vapor pressure can be obtained in the headspace, in spite of the liquid-gas interface moving in the wick during the operation of the calibration source.

Temperature dependence of the generation rate has been measured as well. The result is shown in Fig. 5(b). The experimental data follow the trend predicted by the classic diffusion model. However, the measured generation rates are much higher than the theoretically predicted ones. Since the difference between the two sets of data is almost temperature independent, it is most likely caused by a calibration shift of the FID, but has not yet been experimentally corroborated.

\section{Conclusions}

A new vapor calibration source has been fabricated and tested to provide longer lifetime. Macro PS with straight pores was used as a wick for a liquid reservoir to provide stable vapor generation over an extended time. This vapor source can be easily integrated into a $\mu \mathrm{GC}$ system and is suitable for on-board calibration.

Acknowledgement This work was supported by the National Science Foundation under Award Number EEC9986866 as part of the Engineering Research Center for Wireless Integrated Micro Systems (WIMS).

\section{References}

[1] E. T. Zellers, K. D. Wise, K. Najafi, D. Aslam, R. B. Brown, Q. Y. Cai，J. Driscoll， M. Flynn, J. Giachino, R. Gordenker, M. D. Hsieh, C. T.-C. Nguyen, P. Bergstrom, J. Drelich, C. Friedrich, E. Gamble, M. Kaviany, C. J. Lu, A. Matzger, M. Oborny, S. Pang, J. Potkay, R. Sacks, W.-C. Tian, W. Steinecker, J. Whiting, and Q. Zhong, Technical Digest of the IEEE Conference on Technologies for Homeland Security (Boston, 2002), p. 92.

[2] S. C. Terry, J. H. Jerman, and J. B. Angell, IEEE Trans. Electron Devices 26, 1880 (1979).

[3] M. W. Bruns, Proceedings of 1992 International Conference on Industry Electronics, Control, Instrumentation and Automation, Nov. 1992, pp. 1640-1644.

[4] R. R. Reston and E. S. Kolesar, J. Microelectromech. Syst. 3, 134 (1994).

[5] G. Wiranto, M. R. Haskard, D. E. Mulcahy, D. E. Davey, and E. F. Dawes, Proceedings of 1999 SPIE Conference on Electronics and Structures for MEMS, Washington, USA, Oct. 1999, pp. 168-177.

[6] M. C. Oborny, J. Zheng, J. M. Nichols, C.-J. Lu, P. L. Bergstrom, R. P. Manginell, G. C. Frye-Mason, and E. T. Zellers, $\mu$ TAS2003, Squaw Valley, USA (2003).

[7] C. R. Friedrich and P. J. Coane, J. Microelectronic Engineering 35, 367 (1997).

[8] J. Zheng, M. Christophersen, and P. L. Bergstrom, phys. stat. sol. (a) 202(8), 1402 (2005). 